\title{
Hofstadter's butterfly gets caught in graphene
}

- Hofstadters butterfly in Moiré superlattices: A fractal quantum Hall effect, C. R. Dean, L. Wang, P. Maher, C. Forsythe, F. Ghahari, Y. Gao, J. Katoch, M. Ishigami, P. Moon, M. Koshino, T. Taniguchi, K. Watanabe, K. L. Shepard, J. Hone, and P. Kim, arXiv:1212.4783.

- Changes in Fermi surface topology and Hofstadter quantization in graphene superlattices, L. A. Ponomarenko, R. V. Gorbachev, D. C. Elias, G. L. Yu, A. S. Mayorov, J. Wallbank, M. Mucha-Kruczynski, A. Patel, B. A. Piot, M. Potemski, I. V. Grigorieva, K. S. Novoselov, F. Guinea, V. I. Falko, and A. K. Geim, arXiv:1212.5012.

Recommended with a commentary by Carlo Beenakker, Leiden University

Hofstadter's butterfly has been a holy grail of mesoscopic physics since the 1980's. The self-similar electronic energy spectrum of a two-dimensional lattice in a magnetic field, reminiscent of the fractal pattern of a butterfly wing, was an exercise in mathematical physics when Douglas Hofstadter described it in 1976. The structure appears because of a commensurability of the lattice constant $a$ and the magnetic length $l_{m}=\sqrt{\hbar / e B}$, which for atomic-scale lattices would require impossibly large fields of thousands of Tesla. The mesoscopic scale of quantum dots and superlattices brought the field scale down by several orders of magnitude, and many research groups searched for signatures of the minigaps that the periodic potential should open within a broadened Landau level.

The search remained largely inconclusive, as evidenced by the titles of some of the experimental reports: "chasing the Hofstadter butterfly", "a glimpse of Hofstadter's butterfly" — a 2004 PRL [92, 256801] from the Stuttgart group gave perhaps the strongest evidence for Landau level substructure in a GaAs/AlGaAs heterostructure. The commensurability effects on the magnetoconductance that are easiest to observe, the socalled Weiss oscillations, involve not the magnetic length but the cyclotron radius $m v_{F} / e B$. These are classical rather than quantum effects of a magnetic field (no $\hbar$ enters in the commensurability condition). The quantum effects are very sensitive to lattice inhomogeneities, and since the two-dimensional electron gas is buried $100 \mathrm{~nm}$ inside the heterostructure, it is very difficult to accurately transfer a periodic potential much below the micrometer scale.

Enter graphene. The conduction electrons in the carbon $\pi$-orbitals are directly exposed to the substrate, allowing for the creation of a highly homogeneous periodic potential by the Moiré effect: If the substrate has the same honeycomb lattice as graphene, but slightly misaligned or with a slightly different lattice constant, then the the two lattice structures interfere to produce 
a superlattice with triangular symmetry. For graphene on boron nitride the periodicity of the superlattice is about $10 \mathrm{~nm}$, equal to the magnetic length at 5 Tesla - an ideal range to search for quantum commensurability effects.

Last December two groups reported the results of this search. Andre Geim's group (Manchester University) used monolayer graphene, while Philip Kim's group (Columbia University) used bilayer graphene, with similar findings. A vanishing of the longitudinal conductance $\sigma_{x x}$ with quantized transverse (Hall) conductance $\sigma_{x y}$ was used as a probe of the opening of minigaps within a Landau level. The minigaps are largest if the flux $\phi$ through a unit cell (in units of $h / e$ ) is close to a rational number $p / q$, with small integers $p, q$. The resulting energy spectrum (see figure 7 of arXiv:cond-mat/0607096) repeats in a self-similar way. The zero-field region where $\sigma_{x y}$ changes sign repeats at nonzero field (figure $4 \mathrm{~b}$ of arXiv:1212.4783). Most strikingly, in the monolayer graphene experiments a clone of the Dirac point appears away from charge neutrality (figure 2 of arXiv:1212.5012).

The Dirac point in monolayer graphene is special, because it supports a zero mode - a Landau level that stays pinned at that energy $(E=0)$, while the other Landau levels move up or down with increasing magnetic field. A periodic potential opens a gap at the Dirac point, but the gap closes again and the zero mode reappears for a range of magnetic fields around $\phi=1 / q$. This is the same commensurability effect that produces the self-similar Hofstadter butterfly, but now for massless Dirac electrons. Theoretical simulations have predicted a variety of other features of the Dirac-Hofstadter spectrum, that are now accessible for experimental observation, such as the reappearance of the zero-field conical dispersion at finite magnetic fields.

The experiments are also a timely contribution to the emerging field of topological insulators. The quantization of the Hall conductance $\sigma_{x y}=$ $Q \times e^{2} / h$ in Hofstadter's butterfly is determined by a topological quantum number $Q$ (called Chern number or TKNN invariant), computed as an integral of Bloch states over the Brillouin zone. In the original quantum Hall effect, $Q$ is more mundanely determined by the number of occupied Landau levels, without requiring notions of topology. Haldane's quantum Hall effect without Landau levels, observed very recently in a magnetic topological insulator (Science DOI 10.1126/science.1234414), is another variation on the quantum Hall effect where the topological origin of the quantization becomes explicit. 